\title{
Max-Min Transmit Beamforming via Iterative Regularization
}

\author{
Ahmad Gharanjik ${ }^{\ddagger}$, Bhavani Shankar M. R..$^{\ddagger}$, Mojtaba Soltanalian ${ }^{\dagger}$, and Björn Ottersten ${ }^{* \ddagger}$ \\ $\ddagger$ SnT Centre, University of Luxembourg, ${ }^{*}$ Signal Processing Department, KTH Royal Institute of Technology, \\ $\dagger$ Department of Electrical and Computer Engineering, University of Illinois at Chicago
}

\begin{abstract}
This paper introduces an iterative optimization framework to tackle the multi-group multicast Max-Min transmit beamforming problem. In each iteration, the optimization problem is decomposed into four sub-problems, all of which can be solved using computationally efficient algorithms. The advantage of proposed method lies in its ability to handle different types of signal constraints like total power and unimodularity; a feature not exhibited by other techniques. The proposed technique outperforms the well-known semidefinite relaxation method in terms of quality of solutions.
\end{abstract}

\section{INTRODUCTION}

Max-Min transmit beamforming is a classical design problem in wireless communications [1]-[3], with the goal of maximizing the minimal performance of a network to achieve fairness. In wireless communications, Signal to Noise and Interference Ratio (SINR) of users is widely used as the performance measure and the Max-Min transmit beamforming takes the form

$$
\max _{\left\{\boldsymbol{w}_{i}\right\}} \quad \min _{i} \mathrm{SINR}_{i}
$$

where $\boldsymbol{w}_{i}$ is the beamforming vector for $i^{\text {th }}$ user and $\operatorname{SINR}_{i}$ is the corresponding SINR. This problem was solved for the first time considering the unicast scenario and a total transmit power constraint in [1]. In unicast scenario, independent signals are transmitted to each user. This problem was then solved for multicast set-up in [4] where a common signal is transmitted to all users. The multi-group multicast beamforming design problem as a generalization of the unicast and multicast beamforming problems was studied in [5]. Since then, multi-group multicast beamforming was extended to consider different type of constraints [6], [7]. The Rate-Splitting (RS) concept was introduced to multi-group multicasting and its performance gain was studied in [8].

The optimization problem leading to the design of beamformers is, in general, a NP-hard problem. Most of the algorithms developed to tackle this problem are either based on semidefinite relaxation (SDR) technique, which are computationally expensive, or catering towards specific type of signal constraints, e.g. a total power constraint. Relevant constraints like the unimodularity, which can have applications in phaseonly beamforming [9] and hybrid analog/digital beamforming [10], are not considered by these algorithms. The perceived shortcomings of existing algorithms in terms of complexity

Corresponding author- Email: ahmad.gharanjik@uni.lu and applicability, motivate the exercise carried out in this paper.

In this work, we consider the multi-group multicasting since it can be seen as a generalization of beamforming problems and propose a computationally efficient framework to tackle the corresponding optimization problem. The proposed method is based on iterative paradigm involving the use of regularization based optimization approach. While the proposed framework can efficiently handle several constraints including total power and unimodularity constraints, it also improves on the complexity of related iterative Grab and Pull approach [11].

The paper is organized as follows: in Section II, the system model and problem formulation are presented. In Section III, the optimization problem is reformulated in order to make it tractable. Then, the proposed framework is introduced in Section IV to tackle the optimization problem. Comparison with the SDR technique and numerical results presented in Section V. Finally, Section VI concludes the paper.

\section{System Model ANd PRoblem Formulation}

We consider a transmitter equipped with $N$ antennas that communicates with $M$ single-antenna users distributed in $G$ groups. The users in each group are interested in receiving a common signal. For the special cases of $G=1$ and $G=$ $M$, this scenario coincides with the multicast beamforming and traditional unicast beamforming problems, respectively. We denote the subset of user indices in the $k^{\text {th }}$ group by $\mathcal{G}_{k}$ for any $k \in[G]$. Let $\boldsymbol{h}_{i} \in \mathbb{C}^{N}$ denotes the channel between the transmit antennas and the $i^{\text {th }}$ user, and $\boldsymbol{w}_{k}$ denotes the beamforming vector for $k^{\text {th }}$ group of users. Then the SINR of the $i^{\text {th }}$ user can be written as

$$
\operatorname{SINR}_{i}=\frac{\boldsymbol{w}_{k}^{H} \boldsymbol{R}_{i} \boldsymbol{w}_{k}}{\left(\sum_{j \in[G] \backslash k} \boldsymbol{w}_{j}^{H} \boldsymbol{R}_{i} \boldsymbol{w}_{j}\right)+\sigma_{i}^{2}}, \quad \forall i \in[M],
$$

where $\boldsymbol{R}_{i}=\mathbb{E}\left\{\boldsymbol{h}_{i} \boldsymbol{h}_{i}^{H}\right\}$ is the covariance matrix of the $i^{\text {th }}$ channel and $\sigma_{i}^{2}$ denotes the variance if the zero-mean additive white Gaussian noise (AWGN). Then the problem of maximizing the minimum $\operatorname{SINR}_{i}$ in the network can be 
formulated as [5],

$$
\begin{aligned}
\max _{\left\{\boldsymbol{w}_{k}\right\}_{k=1}^{G}} & \min _{k \in[G]}\left\{\min _{i \in \mathcal{G}_{k}}\left\{\frac{\boldsymbol{w}_{k}^{H} \boldsymbol{R}_{i} \boldsymbol{w}_{k}}{\left(\sum_{j \in[G] \backslash k} \boldsymbol{w}_{j}^{H} \boldsymbol{R}_{i} \boldsymbol{w}_{j}\right)+\sigma_{i}^{2}}\right\}\right\} \\
\text { s. t. } & \sum_{k=1}^{G}\left\|\boldsymbol{w}_{k}\right\|^{2} \leq P,
\end{aligned}
$$

where $P$ is the total transmit power constraint. We define a stacked beamforming vector $\boldsymbol{w} \in \mathbb{C}^{K}$ ( with $K=N G$ ) as $\boldsymbol{w}=\operatorname{vec}\left(\left[\boldsymbol{w}_{i}, \boldsymbol{w}_{2}, \cdots, \boldsymbol{w}_{G}\right]\right)$. It can be shown that the power constraint in (3) will be satisfied with equality, so we have $\|\boldsymbol{w}\|^{2}=P$. Following [11], the SINR expression can be written as

$$
\operatorname{SINR}_{i}=\frac{\boldsymbol{w}^{H} \boldsymbol{A}_{i} \boldsymbol{w}}{\boldsymbol{w}^{H} \boldsymbol{B}_{i} \boldsymbol{w}}, \quad \forall i \in[M],
$$

where $\boldsymbol{A}_{i}$ and $\boldsymbol{B}_{i}$ are positive semidefinite (PSD) matrices, defined as

$$
\begin{aligned}
& \boldsymbol{A}_{i} \triangleq \operatorname{Diag}\left(\boldsymbol{e}_{i}\right) \otimes \boldsymbol{R}_{i}, \quad \forall i \in[M], \\
& \boldsymbol{B}_{i} \triangleq\left(\boldsymbol{I}_{N}-\operatorname{Diag}\left(\boldsymbol{e}_{i}\right)\right) \otimes \boldsymbol{R}_{i}+\frac{\sigma_{i}^{2}}{P} \boldsymbol{I}_{K}, \quad \forall i \in[M] .
\end{aligned}
$$

The Max-Min beamforming design problem can then be recast as the following optimization problem:

$$
\mathcal{P}: \max _{\boldsymbol{w} \in \mathbb{C}^{K}} \min _{i \in[M]}\left\{\frac{\boldsymbol{w}^{H} \boldsymbol{A}_{i} \boldsymbol{w}}{\boldsymbol{w}^{H} \boldsymbol{B}_{i} \boldsymbol{w}}\right\}
$$$$
\text { s. t. } \boldsymbol{w} \in \boldsymbol{\Omega} \text {, }
$$

where $\Omega$ is the feasible set of the problem governed by the constraint on $\boldsymbol{w}$. Particularly, the total power constraint and unimodular signal design, $\boldsymbol{\Omega}$ is defined respectively as [11], [12],

$$
\begin{aligned}
& \boldsymbol{\Omega}=\left\{\boldsymbol{w}:\|\boldsymbol{w}\|^{2}=P\right\}, \\
& \boldsymbol{\Omega}=\left\{e^{j \varphi}: \varphi \in[0,2 \pi)\right\}^{K} .
\end{aligned}
$$

The optimization problem $\mathcal{P}$ is known to be NP-hard in general [4], [5] and several approaches (albeit mostly suboptimal) have been proposed in the literature to solve special forms of $\mathcal{P}$; e.g. for total power constraint (8a) in [5], for multicast scenario $(G=1)$ in [4], for $G=M$ in [1]-[3], [13]. However, these algorithms either require high computational cost towards finding a feasible solution [4], [5] or can not be extended to solve the general form of $\mathcal{P}[1]-[3]$, [13]. The proposed approach herein is computationally efficient and can handle different types of signal constraints. In the next section, we reformulate this problem to facilitate the optimization framework.

\section{PROBLEM REFORMULATION}

We make use of the auxiliary variables $\left\{\gamma_{i}\right\}$, defined as

$$
\gamma_{i}=\frac{\boldsymbol{w}^{H} \boldsymbol{A}_{i} \boldsymbol{w}}{\boldsymbol{w}^{H} \boldsymbol{B}_{i} \boldsymbol{w}} .
$$

The optimization problem $\mathcal{P}$ can then be rewritten as,

$$
\begin{aligned}
\mathcal{P}_{1}: \max _{\boldsymbol{w},\left\{\gamma_{i}\right\}} & \min _{i \in[M]}\left\{\gamma_{i}\right\} \\
\text { s. t. } & \gamma_{i}=\frac{\boldsymbol{w}^{H} \boldsymbol{A}_{i} \boldsymbol{w}}{\boldsymbol{w}^{H} \boldsymbol{B}_{i} \boldsymbol{w}}, \forall i \in[M] \\
& \boldsymbol{w} \in \boldsymbol{\Omega},
\end{aligned}
$$

Denote the minimum of $\left\{\gamma_{i}\right\}$ by $\gamma$, i.e. $\gamma=\min \left\{\gamma_{i}\right\}$. Since $\gamma_{i} \geq 0$, by introducing new slack variables $\left\{t_{i}\right\}$, we have that

$$
\gamma_{i}=\gamma+t_{i}, \quad \gamma \geq 0, t_{i} \geq 0, \quad \forall i \in[M] .
$$

Therefore, we can propose the following equivalent optimization problem to $\mathcal{P}_{1}$ :

$$
\begin{aligned}
\mathcal{P}_{2}: \max _{\boldsymbol{w}, \gamma,\left\{t_{i}\right\}} & \gamma \\
\text { s. t. } & \gamma+t_{i}=\frac{\boldsymbol{w}^{H} \boldsymbol{A}_{i} \boldsymbol{w}}{\boldsymbol{w}^{H} \boldsymbol{B}_{i} \boldsymbol{w}}, \quad \forall i \in[M] \\
& \boldsymbol{w} \in \boldsymbol{\Omega}, t_{i} \geq 0 .
\end{aligned}
$$

It is straightforward to verify that at the optimum of $\mathcal{P}_{2}$, at least one of $\left\{t_{i}\right\}$ should be zero. Since $\boldsymbol{A}_{i}$ and $\boldsymbol{B}_{i}$ are PSD, we can equivalently write the constraints in $\mathcal{P}_{2}$ as

$$
\left\|\boldsymbol{A}_{i}^{\frac{1}{2}} \boldsymbol{w}\right\|=\sqrt{\gamma+t_{i}}\left\|\boldsymbol{B}_{i}^{\frac{1}{2}} \boldsymbol{w}\right\|, \quad \forall i \in[M] .
$$

Using quadratic penalty term, we can write a regularized version of $\mathcal{P}_{2}$ as

$$
\begin{array}{cc}
\mathcal{P}_{3}: \max _{\boldsymbol{w}, \gamma,\left\{t_{i}\right\}} & \gamma-\eta \sum_{i=1}^{M}\left(\left\|\boldsymbol{A}_{i}^{\frac{1}{2}} \boldsymbol{w}\right\|-\sqrt{\gamma+t_{i}}\left\|\boldsymbol{B}_{i}^{\frac{1}{2}} \boldsymbol{w}\right\|\right)^{2} \\
\text { s. t. } & \boldsymbol{w} \in \boldsymbol{\Omega}, \gamma \geq 0, t_{i} \geq 0, \forall i \in[M],
\end{array}
$$

where $\eta$ is the weight of penalty term and $\mathcal{P}_{2}$ and $\mathcal{P}_{3}$ coincide as $\eta \rightarrow \infty$. $\mathcal{P}_{3}$ is still difficult to tackle since the objective will be quartic in $\boldsymbol{w}$. Therefore, by introducing unitary matrix $\boldsymbol{Q}_{i}$, we use another formulation:

$$
\begin{aligned}
\mathcal{P}_{4}: \max _{\boldsymbol{w}, \gamma,\left\{t_{i}\right\},\left\{\boldsymbol{Q}_{i}\right\}} & \gamma-\eta \sum_{i=1}^{M}\left\|\boldsymbol{A}_{i}^{\frac{1}{2}} \boldsymbol{w}-\sqrt{\gamma+t_{i}} \boldsymbol{Q}_{i} \boldsymbol{B}_{i}^{\frac{1}{2}} \boldsymbol{w}\right\|^{2} \\
\text { s. t. } & \boldsymbol{w} \in \boldsymbol{\Omega}, \gamma \geq 0, t_{i} \geq 0 \\
& \boldsymbol{Q}_{i} \in \boldsymbol{U}, \forall i \in[M] .
\end{aligned}
$$

Herein, $\boldsymbol{U}$ is a set of unitary matrices that isometrically transform $\boldsymbol{B}_{i}^{\frac{1}{2}} \boldsymbol{w}$ to the direction of $\boldsymbol{A}_{i}^{\frac{1}{2}} \boldsymbol{w}$; in other words,

$$
\boldsymbol{Q}_{i} \boldsymbol{B}_{i}^{\frac{1}{2}} \boldsymbol{w}=\frac{\left\|\boldsymbol{B}_{i}^{\frac{1}{2}} \boldsymbol{w}\right\|}{\left\|\boldsymbol{A}_{i}^{\frac{1}{2}} \boldsymbol{w}\right\|} \boldsymbol{A}_{i}^{\frac{1}{2}} \boldsymbol{w} .
$$

It is straightforward to verify that by choosing optimal $\boldsymbol{Q}_{i}$ that satisfies (14), formulations $\mathcal{P}_{4}$ and $\mathcal{P}_{3}$ will be equivalent and hence will be used interchangeably. Formulation $\mathcal{P}_{4}$ leads to quadratic objective function w.r.t. $\boldsymbol{w}$ which is easier to tackle.

\section{OPTIMIZATION FRAMEWORK}

In this section, we introduce the proposed optimization framework to tackle $\mathcal{P}_{4}$ (and $\mathcal{P}_{3}$ ). The framework is based on separate optimization of the objective of $\mathcal{P}_{4}$ and $\mathcal{P}_{3}$ over its variables $\boldsymbol{w},\left\{\boldsymbol{Q}_{i}\right\},\left\{t_{i}\right\}$, and $\gamma$ at each iteration 


\section{A. Optimization w.r.t. $\boldsymbol{w}$}

For fixed $\left\{\boldsymbol{Q}_{i}\right\},\left\{t_{i}\right\}$, and $\gamma$, we can show that optimizing the objective of $\mathcal{P}_{4}$ w.r.t. $\boldsymbol{w}$ is equivalent to minimizing the following term,

$$
\sum_{i=1}^{M}\left\|\boldsymbol{A}_{i}^{\frac{1}{2}} \boldsymbol{w}-\sqrt{\gamma+t_{i}} \boldsymbol{Q}_{i} \boldsymbol{B}_{i}^{\frac{1}{2}} \boldsymbol{w}\right\|^{2}=\boldsymbol{w}^{H} \boldsymbol{R} \boldsymbol{w}
$$

where

$$
\boldsymbol{R}=\sum_{i=1}^{M}\left\{\boldsymbol{A}_{i}+\left(\gamma+t_{i}\right) \boldsymbol{B}_{i}-\sqrt{\gamma+t_{i}}\left(\boldsymbol{A}_{i}^{\frac{1}{2}} \boldsymbol{Q}_{i} \boldsymbol{B}_{i}^{\frac{1}{2}}+\boldsymbol{B}_{i}^{\frac{1}{2}} \boldsymbol{Q}_{i}^{H} \boldsymbol{A}_{i}^{\frac{1}{2}}\right)\right\}
$$

To minimize $\boldsymbol{w}^{H} \boldsymbol{R} \boldsymbol{w}$, we can equivalently maximize $\boldsymbol{w}^{H}(-\boldsymbol{R}) \boldsymbol{w}$. For total power constraint, this optimization problem can be solved efficiently using power-method [11]. The unimodular signal design can be handled by using powermethod like iterations introduced in [12]. For more details on handling optimization w.r.t $\boldsymbol{w}$ please refer to [11].

\section{B. Optimization w. r. t. $\gamma$}

Assuming $\left\{\boldsymbol{Q}_{i}\right\},\left\{t_{i}\right\}$ and $\boldsymbol{w}$ are given, we can show that optimization of $\mathcal{P}_{3}$ w. r. t. $\gamma$ can be written as

$$
\max _{\gamma \geq 0} . f(\gamma)
$$

where

$$
f(\gamma)=\gamma-\eta \sum_{i=1}^{K}\left(\alpha_{i}-\sqrt{\gamma+t_{i}} \beta_{i}\right)^{2}
$$

and $\alpha_{i}=\left\|\boldsymbol{A}_{i}^{\frac{1}{2}} \boldsymbol{w}\right\|_{2}$ and $\beta_{i}=\left\|\boldsymbol{B}_{i}^{\frac{1}{2}} \boldsymbol{w}\right\|_{2}$. It is straightforward to find the second derivative of $f(\lambda)$ as

$$
f^{\prime \prime}(\gamma)=-\frac{\eta}{2} \sum_{i=1}^{K} \frac{\alpha_{i} \beta_{i}}{\left(\gamma+t_{i}\right)^{\frac{3}{2}}} .
$$

Note that $f(\gamma)$ is a concave function as $f^{\prime \prime}(\gamma) \leq 0$. Therefore, $-f(\lambda)$ is a convex function and thus the scalar convex optimization problem

$$
\min _{\gamma \geq 0} \cdot-f(\gamma)
$$

can be solved (efficiently) using gradient descent method,

\section{Optimal $\left\{\boldsymbol{Q}_{i}\right\}$}

Now, suppose $\boldsymbol{w},\left\{t_{i}\right\}$ and $\lambda$ are fixed. As discussed earlier, the maximizer $\boldsymbol{Q}_{i}$ is an unitary matrix that maps $\boldsymbol{B}_{i}^{\frac{1}{2}} \boldsymbol{w}$ in the same direction as $\boldsymbol{A}_{i}^{\frac{1}{2}} \boldsymbol{w}$. Matrix $\boldsymbol{Q}_{i}$ can be found using Householder matrix [14] as follows. Let us define $\boldsymbol{u}_{i}=\boldsymbol{B}_{i}^{\frac{1}{2}} \boldsymbol{w}$ and denote the direction of $\boldsymbol{A}_{i}^{\frac{1}{2}} \boldsymbol{w}$ by a unit norm vector $\boldsymbol{v}_{i}$ as

$$
\boldsymbol{v}_{i}=\boldsymbol{A}_{i}^{\frac{1}{2}} \boldsymbol{w} /\left\|\boldsymbol{A}_{i}^{\frac{1}{2}} \boldsymbol{w}\right\| .
$$

Therefore matrix $\boldsymbol{Q}_{i}$ should rotate $\boldsymbol{u}_{i}$ to the direction of $\boldsymbol{v}_{i}$, that is $\boldsymbol{Q}_{i} \boldsymbol{u}_{i}=\left\|\boldsymbol{u}_{i}\right\| \boldsymbol{v}_{i}$. Now, let us define vector $\boldsymbol{z}_{i}$ as

$$
\boldsymbol{z}_{i}=\frac{\boldsymbol{u}_{i}-\left\|\boldsymbol{u}_{i}\right\| \boldsymbol{v}_{i}}{\left\|\left(\boldsymbol{u}_{i}-\left\|\boldsymbol{u}_{i}\right\| \boldsymbol{v}_{i}\right)\right\|} .
$$

Then $\boldsymbol{Q}_{i}$ can be found as

$$
\boldsymbol{Q}_{i}=\boldsymbol{I}-2 \boldsymbol{z}_{i} \boldsymbol{z}_{i}^{H} .
$$

It is straightforward to verify that $\boldsymbol{Q}_{i} \boldsymbol{u}_{i}=\left\|\boldsymbol{u}_{i}\right\| \boldsymbol{v}_{i}$.

\section{Optimization w. r. t. $\left\{t_{i}\right\}$}

For fixed $\boldsymbol{w},\left\{\boldsymbol{Q}_{i}\right\}$ and $\gamma$, values of $\left\{t_{i}\right\}$ can be simply found using the constraint in $\mathcal{P}_{2}$ :

$$
t_{i}=\frac{\boldsymbol{w}^{H} \boldsymbol{A}_{i} \boldsymbol{w}}{\boldsymbol{w}^{H} \boldsymbol{B}_{i} \boldsymbol{w}}-\gamma .
$$

Since there is a non-negativity constraint on $t_{i}$ as well, $t_{i} \geq 0$, the optimal values are given by

$$
t_{i}^{\star}= \begin{cases}t_{i} & \text { if } t_{i} \geq 0 \\ 0 & \text { if } t_{i}<0\end{cases}
$$

\section{Numerical Results}

In this section, in order to evaluate the performance of the proposed algorithm, we compare it with the widely used semidefinite relaxation (SDR) approach [15]. We consider a downlink transmitter with $N=4$ antennas, as well as $M=12$ single-antenna users which are divided into $G=2$ multicast groups of 6 users each. The entries of the channel vectors $\boldsymbol{h}_{i}$ are drawn from an i.i.d. complex Gaussian distribution with zero-mean and unit-variance. The Gaussian noise components received at each user antenna are assumed to have unit variance, i.e. $\sigma_{i}^{2}=1$ for all $i \in[K]$. We consider a normalized total-power constraint, i.e. with $P=1$, and terminate the optimization iterations whenever the objective increase becomes bounded by $\epsilon=10^{-6}$.

\section{A. Comparison with SDR Method}

Considering the total power constraint on the signal $\left(\|\boldsymbol{w}\|^{2}=1\right), \mathcal{P}_{1}$ can be equivalently reformulated as

$$
\begin{aligned}
\mathcal{R}_{1}: \max _{\boldsymbol{W}} . & \min _{i \in[M]}\left\{\frac{\operatorname{Tr}\left(\boldsymbol{A}_{i} \boldsymbol{W}\right)}{\operatorname{Tr}\left(\boldsymbol{B}_{i} \boldsymbol{W}\right)}\right\} \\
\text { s. t. } & \operatorname{Tr}(\boldsymbol{W})=1, \boldsymbol{W} \succeq \mathbf{0}, \operatorname{rank}(\boldsymbol{W})=1,
\end{aligned}
$$

where $\boldsymbol{W}=\boldsymbol{w} \boldsymbol{w}^{H}$. Relaxing the rank-one constraint and noting that the objective function is quasi-concave, we can write the corresponding feasibility problem as follows:

$$
\begin{aligned}
& \mathcal{R}_{2} \text { : find } \boldsymbol{W} \\
& \text { s. t. } \frac{\operatorname{Tr}\left(\boldsymbol{A}_{i} \boldsymbol{W}\right)}{\operatorname{Tr}\left(\boldsymbol{B}_{i} \boldsymbol{W}\right)} \geq \mu, \quad \forall i \in[M], \\
& \operatorname{Tr}(\boldsymbol{W})=1, \boldsymbol{W} \succeq \mathbf{0} .
\end{aligned}
$$

Note that a maximal value of $\mu$ may be found using the bisection method. Particularly, $\mathcal{R}_{2}$ followed by the bisection procedure is equivalent to a relaxed version of $\mathcal{R}_{1}$ in which the rank-one constraint is dropped. Moreover, for any given $\mu$, $\mathcal{R}_{2}$ is a (convex) semidefinite program and can be solved using interior-point solvers [15]. We stop the bisection in the solver whenever the increments in $\mu$ become bounded by $10^{-6}$.

Let $\boldsymbol{W}^{\star}$ denote the solution to $\mathcal{R}_{2}$ after the bisection procedure is complete. As $\mathcal{R}_{2}$ followed by bisection is a relaxed version of $\mathcal{R}_{1}$ (and $\mathcal{P}_{1}$ ), the optimal value of its objective yields an upper bound on the optimal objectives of 
TABLE I

COMPARISON OF THE PERFORMANCE OF THE PROPOSED METHOD AND SDR IN MULTI-GROUP MULTICASTING FOR 300 RANDOM REALIZATION OF THE CHANNEL $(N=8, K=12)$.

\begin{tabular}{|c|c|l|l|l|l|}
\hline$K$ & $\eta$ & $\begin{array}{l}\text { Average } \\
\gamma^{\star} / v_{\mathrm{SDR}}\end{array}$ & $\begin{array}{l}\text { Average } \\
\gamma^{\star} / v_{\mathrm{SDR}}^{\star}\end{array}$ & $\begin{array}{l}\text { Average } \\
\mathrm{CPU} \\
(\mathrm{sec})\end{array}$ & $\begin{array}{l}\text { Average SDR } \\
\text { time / Average } \\
\text { CPU time }\end{array}$ \\
\hline \multirow{3}{*}{$\mathbf{1 2}$} & $\mathbf{1}$ & 1.16 & 0.78 & 0.28 & 7.5 \\
\cline { 2 - 6 } & $\mathbf{1 0}$ & 1.25 & 0.88 & 1.67 & 1.21 \\
\cline { 2 - 6 } & $\mathbf{0 . 5 / 1 0} / \mathbf{1 0 0 0}$ & 1.21 & 0.84 & 0.56 & 3.6 \\
\hline
\end{tabular}
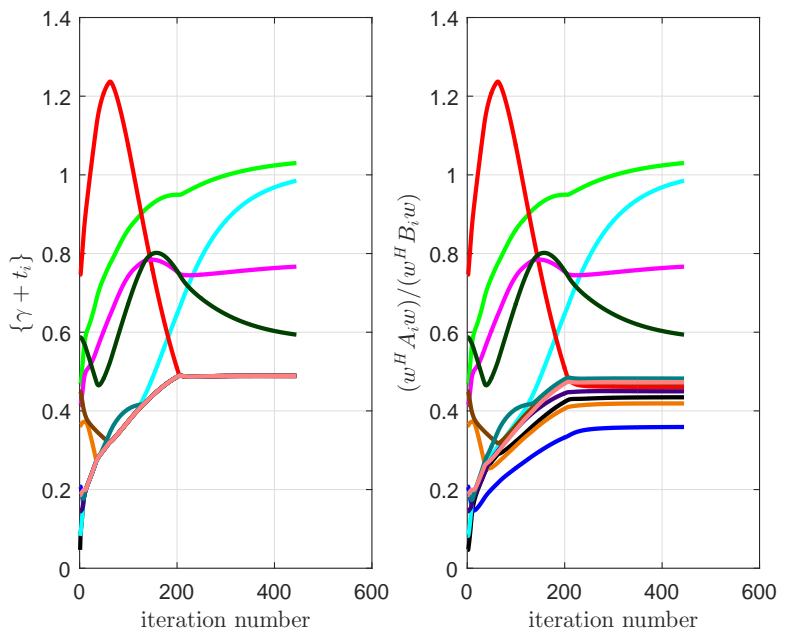

(a)
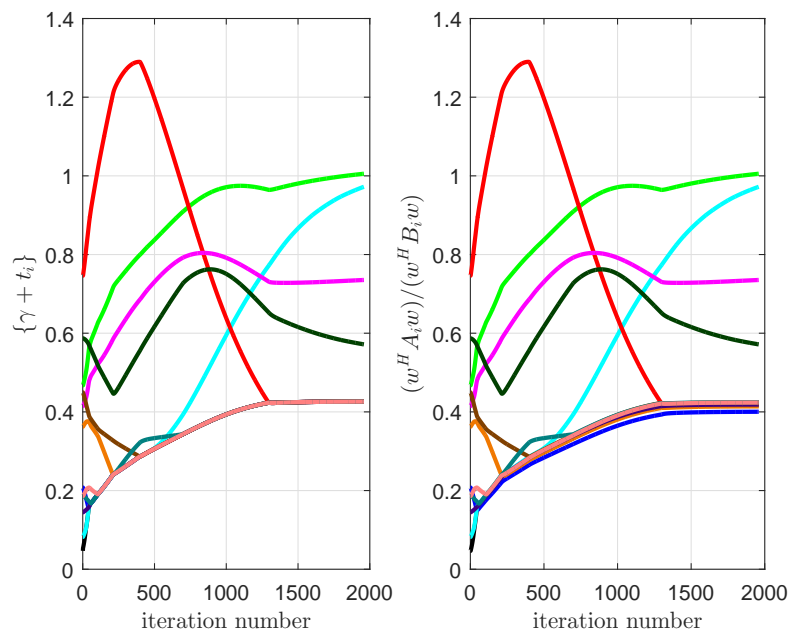

(b)

Fig. 1. Transition of the optimization parameters $\left\{\gamma+t_{i}\right\}$ and $\left(\boldsymbol{w}^{H} \boldsymbol{A}_{i} \boldsymbol{w}\right) /\left(\boldsymbol{w}^{H} \boldsymbol{B}_{i} \boldsymbol{w}\right)$ (distinguished by colors for different $\left.i\right)$ vs. the iteration number for different weights $(\eta)$ of the regularization term in $\mathcal{P}_{3}$ and $\mathcal{P}_{4}$ : (a) $\eta=2$, and (b) $\eta=10$.

$\mathcal{R}_{1}$ (and $\mathcal{P}_{1}$ ), although it may not be tight. This upper bound is given as

$$
v_{\mathrm{SDR}}^{\star}=\min _{i \in[M]}\left\{\frac{\operatorname{Tr}\left(\boldsymbol{A}_{i} \boldsymbol{W}^{\star}\right)}{\operatorname{Tr}\left(\boldsymbol{B}_{i} \boldsymbol{W}^{\star}\right)}\right\} .
$$

which may be used to examine further the goodness of approximate solutions provided by various methods.

Due to the rank relaxation in $\mathcal{R}_{2}, \boldsymbol{W}^{\star}$ will not (in general) be unit rank. In this case, the Gaussian randomization (GR) method [15], [17] is typically used to generate $L$ candidates (from which, the one leading to the largest objective, will be chosen) for approximating the optimum $\boldsymbol{w}^{\star}$ of $\mathcal{P}_{1}$ : Let $\boldsymbol{W}^{\star}=$ $\boldsymbol{V} \boldsymbol{\Sigma} \boldsymbol{V}^{H}$ be the eigen-decomposition of $\boldsymbol{W}^{\star}$. The $l^{\text {th }}$ candidate $(l \in[L])$ can be generated as $\boldsymbol{w}_{l}=\boldsymbol{V} \boldsymbol{\Sigma}^{1 / 2} \boldsymbol{v}_{l}$, where $\boldsymbol{v}_{l} \in$ $\mathbb{C}^{K} \sim \mathcal{C N}(\mathbf{0}, \boldsymbol{I})$ [5]. Note that each $\boldsymbol{w}_{l}$ may be scaled in order to satisfy the constraint $\left\|\boldsymbol{w}_{l}\right\|^{2}=1$. We denote the best candidate by $\boldsymbol{w}_{G R}^{\star}$. The corresponding objective value is thus given by

$$
v_{\mathrm{SDR}}=\min _{i \in[M]}\left\{\frac{\boldsymbol{w}_{G R}^{\star H} \boldsymbol{A}_{i} \boldsymbol{w}_{G R}^{\star}}{\boldsymbol{w}_{G R}^{\star H} \boldsymbol{B}_{i} \boldsymbol{w}_{G R}^{\star}}\right\} .
$$

Table I summarizes the results of Max-Min beamforming design for 300 random realizations of the multi-group multicasting channel. Average performance of the proposed algorithm for different values of $\eta$ is compared with SDR followed by 1000 GRs. It can be seen that $\eta=10$ leads to higher objective of $\mathcal{P}_{1}$ but increases the run-time of the algorithm. On the other hand, increasing $\eta$ in few steps, i.e. the case with $\eta=0.5 / 10 / 1000$, provides a good balance between the solution quality and run-time, while outperforming SDR in both criteria.

Fig. 1 shows the transition of the optimization parameters $\gamma+t_{i}$ and $\left(\boldsymbol{w}^{H} \boldsymbol{A}_{i} \boldsymbol{w}\right) /\left(\boldsymbol{w}^{H} \boldsymbol{B}_{i} \boldsymbol{w}\right)$ vs. the iteration number for (a) $\eta=2$, and (b) $\eta=10$. Note that these parameters are equal as $\eta \rightarrow \infty$ and it can be observed that by increasing $\eta$ from 2 to 10 , difference between $\gamma+t_{i}$ and $\left(\boldsymbol{w}^{H} \boldsymbol{A}_{i} \boldsymbol{w}\right) /\left(\boldsymbol{w}^{H} \boldsymbol{B}_{i} \boldsymbol{w}\right)$ reduces. It should also be noted that $\left.\min \left\{\left(\boldsymbol{w}^{H} \boldsymbol{A}_{i} \boldsymbol{w}\right) /\left(\boldsymbol{w}^{H} \boldsymbol{B}_{i} \boldsymbol{w}\right)\right)\right\}$ is monotonically increasing over the iterations.

\section{CONCLUSION}

A new iterative optimization framework based on penalized reformulation is proposed to tackle the Max-Min beamforming design problem. The proposed approach is computationally very efficient and outperforms the SDR method. It can also handle total power and unimodularity signal constraints. 


\section{REFERENCES}

[1] M. Schubert and H. Boche, "Solution of the multiuser downlink beamforming problem with individual SINR constraints," IEEE Transactions on Vehicular Technology, vol. 53, no. 1, pp. 18-28, Jan. 2004.

[2] A. Wiesel, Y. Eldar, and S. Shamai, "Linear precoding via conic optimization for fixed MIMO receivers," IEEE Transactions on Signal Processing, vol. 54, no. 1, pp. 161-176, Jan. 2006.

[3] D. P. Palomar, J. M. Cioffi, and M. A. Lagunas, "Joint tx-rx beamforming design for multicarrier mimo channels: A unified framework for convex optimization," IEEE Transactions on Signal Processing, vol. 51, no. 9, pp. 2381-2401, 2003.

[4] N. D. Sidiropoulos, T. N. Davidson, and Z.-Q. T. Luo, "Transmit beamforming for physical-layer multicasting," IEEE Transactions on Signal Processing, vol. 54, no. 6, pp. 2239-2251, 2006.

[5] E. Karipidis, N. D. Sidiropoulos, and Z.-Q. Luo, "Quality of service and max-min fair transmit beamforming to multiple cochannel multicast groups," IEEE Transactions on Signal Processing, vol. 56, no. 3, pp. 1268-1279, 2008.

[6] D. Christopoulos, S. Chatzinotas, and B. Ottersten, "Weighted fair multicast multigroup beamforming under per-antenna power constraints," IEEE Transactions on Signal Processing, vol. 62, no. 19, pp. 5132$5142,2014$.

[7] _ - "Multicast multigroup beamforming for per-antenna power constrained large-scale arrays," in 2015 IEEE 16th International Workshop on Signal Processing Advances in Wireless Communications (SPAWC). IEEE, 2015, pp. 271-275.

[8] H. Joudeh and B. Clerckx, "A rate-splitting strategy for max-min fair multigroup multicasting," in 2016 IEEE 17th International Workshop on Signal Processing Advances in Wireless Communications (SPAWC), July 2016, pp. 1-5.

[9] Ö. T. Demir and T. E. Tuncer, "Optimum phase-only discrete broadcast beamforming with antenna and user selection in interference limited cognitive radio networks," in 2015 IEEE International Conference on Acoustics, Speech and Signal Processing (ICASSP). IEEE, 2015, pp. 2724-2728.

[10] A. Alkhateeb, O. El Ayach, G. Leus, and R. W. Heath, "Hybrid precoding for millimeter wave cellular systems with partial channel knowledge," in Information Theory and Applications Workshop (ITA), 2013. IEEE, 2013, pp. 1-5.

[11] M. Soltanalian, A. Gharanjik, B. Shankar, and B. Ottersten, "Grabn-Pull: An optimization framework for fairness-achieving networks," accepted to IEEE International Conference on Acoustics, Speech and Signal Processing, ICASSP, 2016.

[12] M. Soltanalian and P. Stoica, "Designing unimodular codes via quadratic optimization," IEEE Transactions on Signal Processing, vol. 62, no. 5, pp. 1221-1234, March 2014.

[13] G. Dartmann, X. Gong, W. Afzal, and G. Ascheid, "On the Duality of the Max-Min Beamforming Problem With Per-Antenna and Per-AntennaArray Power Constraints," IEEE Transactions on Vehicular Technology, vol. 62, no. 2, pp. 606-619, Feb. 2013.

[14] R. A. Horn and C. R. Johnson, Matrix Analysis, 2nd ed. Cambridge; New York: Cambridge University Press, Dec. 2012.

[15] Z.-Q. Luo, W.-K. Ma, A.-C. So, Y. Ye, and S. Zhang, "Semidefinite relaxation of quadratic optimization problems," IEEE Signal Processing Magazine, vol. 27, no. 3, pp. 20-34, 2010.

[16] CVX Research Inc., "CVX: Matlab software for disciplined convex programming, version 2.0," http://cvxr.com/cvx, Aug. 2012.

[17] S. Zhang and Y. Huang, "Complex quadratic optimization and semidefinite programming," SIAM Journal on Optimization, vol. 16, no. 3, pp. 871-890, 2006. 